\title{
Stereomammography: Evaluation of depth perception using a virtual 3D cursor
}

\author{
Mitchell M. Goodsitt, ${ }^{a}$ ) Heang-Ping Chan, and Lubomir Hadjiiski \\ Department of Radiology, University of Michigan, Ann Arbor, Michigan 48109-0030
}

(Received 22 October 1999; accepted for publication 28 March 2000)

\begin{abstract}
We are evaluating the usefulness of stereomammography in improving breast cancer diagnosis. One area that we are investigating is whether the improved depth perception associated with stereomammography might be significantly enhanced with the use of a virtual 3D cursor. A study was performed to evaluate the accuracy of absolute depth measurements made in stereomammograms with such a cursor. A biopsy unit was used to produce digital stereo images of a phantom containing 50 low contrast fibrils $(0.5 \mathrm{~mm}$ diam monofilaments) at depths ranging from 1 to $11 \mathrm{~mm}$, with a minimum spacing of $2 \mathrm{~mm}$. Half of the fibrils were oriented perpendicular (vertical) and half parallel (horizontal) to the stereo shift direction. The depth and orientation of each fibril were randomized, and the horizontal and vertical fibrils crossed, simulating overlapping structures in a breast image. Left and right eye images were generated by shifting the $\mathrm{x}$-ray tube from $+2.5^{\circ}$ to $-2.5^{\circ}$ relative to the image receptor. Three observers viewed these images on a computer display with stereo glasses and adjusted the position of a cross-shaped virtual cursor to best match the perceived location of each fibril. The $x, y$, and $z$ positions of the cursor were indicated on the display. The $z$ (depth) coordinate was separately calibrated using known positions of fibrils in the phantom. The observers analyzed images of two configurations of the phantom. Thus, each observer made 50 vertical filament depth measurements and 50 horizontal filament depth measurements. These measurements were compared with the true depths. The correlation coefficients between the measured and true depths of the vertically oriented fibrils for the three observers were $0.99,0.97$, and 0.89 with standard errors of the estimates of $0.39 \mathrm{~mm}, 0.83 \mathrm{~mm}$, and $1.33 \mathrm{~mm}$, respectively. Corresponding values for the horizontally oriented fibrils were $0.91,0.28$, and 0.08 , and $1.87 \mathrm{~mm}, 4.19 \mathrm{~mm}$, and $3.13 \mathrm{~mm}$. All observers could estimate the absolute depths of vertically oriented objects fairly accurately in digital stereomammograms; however, only one observer was able to accurately estimate the depths of horizontally oriented objects. This may relate to different aptitudes for stereoscopic visualization. The orientations of most objects in actual mammograms are combinations of horizontal and vertical. Further studies are planned to evaluate absolute depth measurements of fibrils oriented at various intermediate angles and of objects of different shapes. The effects of the shape and contrast of the virtual cursor and the stereo shift angle on the accuracy of the depth measurements will also be investigated. (C) 2000 American Association of Physicists in Medicine. [S0094-2405(00)01406-1]
\end{abstract}

Key words: stereomammography, stereoscopic, virtual cursor, three-dimensional

\section{INTRODUCTION}

Presently, screening x-ray mammography is the only technique that has a proven capability for detecting early stage clinically occult cancers. ${ }^{1}$ Although mammography has a high sensitivity for detecting breast cancers, studies have shown that radiologists do not detect all carcinomas that are visible on retrospective analyses of the mammograms. ${ }^{2-11}$ These missed detections are often a result of the very subtle nature of the mammographic findings. One of the major deficiencies of mammography is the inability to discern masses and microcalcifications hidden in dense fibroglandular tissue. ${ }^{12}$ It is estimated that about $20 \%$ of the breast cancers in dense breasts are not detected by mammography. ${ }^{9,11}$ Conventional mammography is a two-dimensional projection image of a three-dimensional structure. As a result, objects along the same x-ray beam path overlap each other. The overlying tissue structures often obscure the visibility of subtle lesions of interest in the mammogram. The camouflaging of the anatomical structures is the main cause of missed diagnoses. Overlapping structures can also project onto the image plane forming shadows that appear to be lesions, resulting in false positive findings. Radiologists examine two or more projections of each breast to improve their ability to detect lesions and to assist them in distinguishing between true lesions and overlapping tissues. However, standard mammographic techniques are not always successful in distinguishing true lesions from overlapping tissues. Digital stereomammography is a method that could potentially solve many of these problems.

Stereomammography is not a new technique. It was first described in $1930 .{ }^{13}$ Like other forms of stereoradiography at that time, it involved taking two film images, a left eye image and a right eye image. These were obtained by positioning the $\mathrm{x}$-ray tube at a certain distance to the left and to the 
right of the central axis. Usually, the total tube shift was $10 \%$ of the source-to-image distance. ${ }^{14}$ The radiologist would view the images using a cross-eyed technique or a special stereoscopic viewer. ${ }^{14}$ Stereoradiography and stereomammography lost favor because of the increased radiation dose, procedure time, and film costs associated with taking two radiographs, and because it generally took more time to read stereoradiographs. According to Christensen's Physics of Diagnostic Radiology, ${ }^{14}$ another reason for reduced use of stereoradiography was radiologists' disappointment with the technique because they failed to appreciate the fact that stereoradiography did not enable them to accurately judge the distances between objects. Rather, stereoradiography allowed for relative depth perception, whereby one could "accurately rank objects in their order of closeness." 14

The advent of digital imaging techniques and video image displays has made stereoradiography and stereomammography attractive again. Research has been performed in digital stereoangiography ${ }^{15-17}$ and digital stereomammography. ${ }^{18,19}$ Furthermore, stereotaxic techniques have been developed for core biopsies of breast lesions. In stereotaxic breast biopsies, much larger stereo angles $\left(+15^{\circ}\right.$ to the right and $-15^{\circ}$ to the left) are employed, compared to the angles used in stereoscopic visualization. These larger angles result in increased parallax ("the apparent displacement of an object when viewed from two different vantage points", ${ }^{14}$ ) which in turn permits more accurate depth determination. With stereotaxic techniques, the operator identifies the location of the lesion in each image, and a computer calculates the spatial coordinates $[x, y$, and $z$ (depth) $]$ of the lesion using equations derived from simple geometry. ${ }^{20}$ For example the distance of the lesion from a fixed image receptor, $z_{l}$, is given by the equation

$$
z_{l}=x_{l s} /\left(2 \tan \left(15^{\circ}\right)\right),
$$

where $x_{l s}$ is the parallax shift of the lesion on the image receptor. ${ }^{20}$

This capacity to measure absolute depths contradicts the relative depth perception limitation of stereoradiography mentioned by Christensen, and in considering this, we conceived the idea of using a virtual (3D) cursor to determine the positions of lesions within a stereoscopic image. The proposed virtual cursor would be calibrated in the $x, y$, and $z$ directions and would be displayed and moved within the stereoscopic image. To our knowledge, such a cursor has never been developed or used in stereoradiography. We performed a literature search and did find that stereographic cursors or pointers have been developed and tested for other purposes, especially for computer graphics and for the operation of robots in remote environments. ${ }^{21-24}$ The application of these cursors to $\mathrm{x}$-ray images as opposed to video or computer graphic images is quite different because $\mathrm{x}$-ray images result from transmission rather than reflection and therefore have a more cloudlike, transparent/translucent quality. Furthermore, additional depth cues due to perspective (closer objects appearing larger than distant objects), occlusion (closer objects obscuring distant objects), shadows (in particular, interactive shadows that move as the objects' posi-

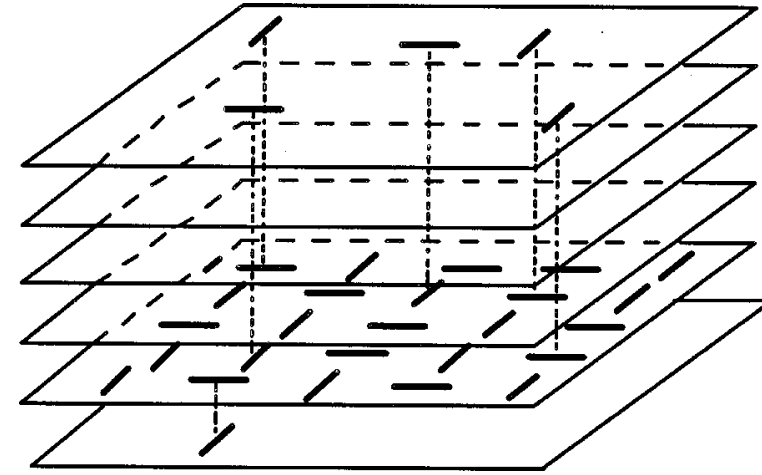

FIG. 1. Diagram of the stereo phantom with simulated fibrils. There are six layers of 1-mm-thick Lexan plates. In the design employed, one of the layers (the second lowest one in this illustration) is superimposed with a $5 \times 5$ array of fibrils randomly oriented in two directions. Each of the other five layers has 5 fibrils placed at randomly chosen locations, with the constraint that no more than 2 fibrils will line up in the same location. The two fibrils at the same location are always oriented perpendicular to each other. For clarity, only 5 fibrils in the top layer and 1 fibril in the bottom layer are drawn. The order of the 6 layers was changed to create the two independent phantoms that were analyzed by each viewer.

tions change), and texture (closer objects having more distinct surface features $)^{25}$ are not apparent in radiographs.

The purpose of the present paper is to describe a proofof-concept study that we performed using a virtual cursor in stereomammography images to determine the depths of selected objects.

\section{METHODS}

\section{A. Phantom}

The phantom that was employed to evaluate the depth accuracy of measurements made with the virtual cursor consisted of six $10-\mathrm{cm} \times 10-\mathrm{cm}$ sheets of 1 -mm-thick Lexan separated by $1-\mathrm{mm}$-thick spacers placed at the corners of each sheet. The test objects were 8-mm long, $0.53-\mathrm{mm}$ diam fibrils [nylon monofilaments (e.g., fish line)], which simulated low contrast spiculations in mammograms. The fibrils were positioned within a $4.5-\mathrm{cm} \times 4.5-\mathrm{cm}$ central region of the Lexan sheets. A total of 50 fibrils were taped to the sheets with 25 oriented perpendicular (vertical) and 25 oriented parallel (horizontal) to the stereo shift direction. The depth and orientation of each fibril were randomized, and the horizontal and vertical fibrils crossed simulating overlapping structures in a breast image. The end result was a $5 \times 5$ array of crossed (horizontal and vertical) filaments, each of which could be examined for its depth (see Fig. 1). With this arrangement, the minimum depth difference between the fibrils was $2 \mathrm{~mm}$ and the maximum was $10 \mathrm{~mm}$. The order of the six Lexan layers could be varied to create many independent phantom configurations for analysis by each reader. In this study, two configurations were randomly chosen.

\section{B. Stereo image acquisition}

The phantom was imaged with a Fischer (Denver, CO) MammoVision Stereotaxic unit. According to Christensen's 

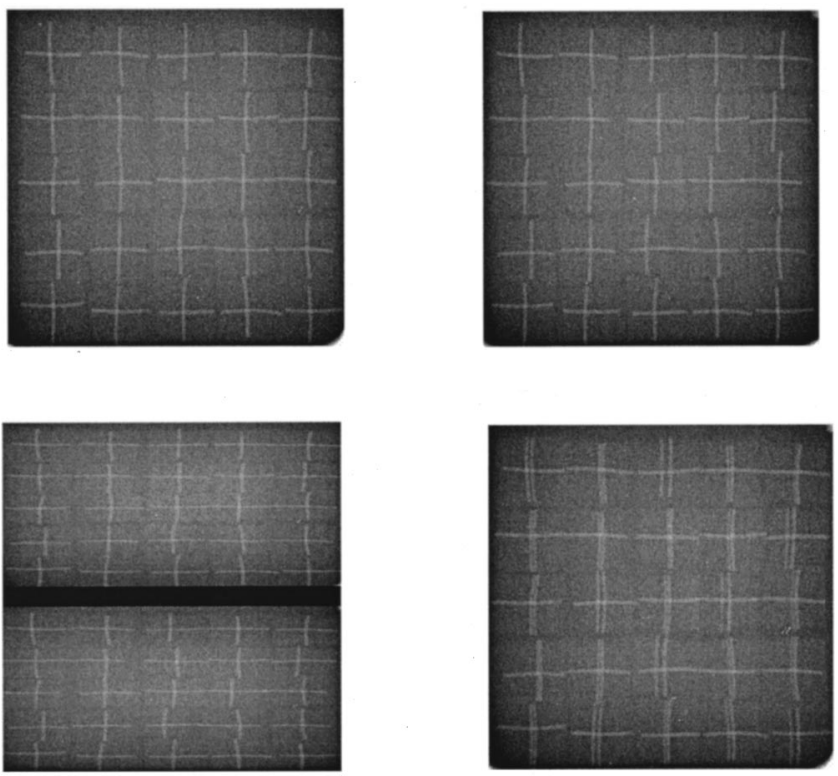

FIG. 2. (Top) Left and right eye images of the phantom shown in Fig. 1. The image pair was obtained with a stereoscopic angle of $\pm 2.5^{\circ}$ about the central axis. The fibril pairs with the smallest spacing of $2 \mathrm{~mm}$ in this phantom can be identified and the relative depths of the different fibrils can also be clearly distinguished. (Bottom left) Image of the phantom that is stored in the computer frame buffer. This image is synch-doubled by the display processor for stereoscopic viewing. Synch-doubling enables viewing of the left- and right-eye images at twice the nominal refresh rate of the display monitor for reduced flicker. The left-eye image is stored in the frame buffer at the top, and the right-eye image at the bottom. An additional vertical synch pulse is inserted between the two images to produce the two separate images shown at the top of this figure. (Bottom right) Image formed by combining the left- and right-eye images into one. An image similar to this is seen when one views the display without the stereoscopic glasses, and is the one used to calibrate the virtual cursor. The virtual cursor (not shown above) appears as two cursors (a left-eye cursor and a right-eye cursor) when the images are viewed without the stereo glasses, and the horizontal separation between the cursors changes as the cursor depth is adjusted.

Physics of Diagnostic Radiology, ${ }^{14}$ early radiologists learned by "trial and error that a tube shift equal to $10 \%$ of the target-film distance produced satisfactory results." 14 This tube shift is equal to a total stereo-shift angle of about $6^{\circ}$ (e.g., $+3^{\circ}$ and $-3^{\circ}$ relative to a line perpendicular to the image receptor.) In general, larger tube shifts produce improved depth perception, but beyond a certain limit, this is achieved at the expense of increased observer fatigue ${ }^{26}$ and decreased stereo field of view. The angle scale on the Fischer unit is marked in $5^{\circ}$ increments, and our preliminary investigations with the Fischer digital system indicated a stereo shift of $+2.5^{\circ}$ to $-2.5^{\circ}$ produced images that appeared to have adequate depth discrimination without producing undue eyestrain. This stereo angle was therefore used for image acquisition in this study. It corresponds with a total stereo shift of about $9 \%(5.94 \mathrm{~cm})$ for the $68 \mathrm{~cm}$ source-to-image distance of the Fischer system. Future studies will be performed to determine the optimal angle for accurate depth perception with acceptable eyestrain. The Fischer unit has a fiber optic-coupled CCD detector that produces $1024 \times 1024$ $\times 12$-bit images. The images can be stored in $1024 \times 1024$ $\times 8$-bit TIFF format or transmitted to a DICOM server. We
TABLE I. Linear regression results for measured vs true depths in phantom images. (A) Vertically oriented fibrils. (B) Horizontally oriented fibrils.

\begin{tabular}{|c|c|c|c|c|c|}
\hline Reader & Image & Slope & Intercept & $r$-value & SEE (mm) \\
\hline \multicolumn{6}{|l|}{ (A) } \\
\hline$A$ & 1 & 1.025 & -0.56 & 0.992 & 0.39 \\
\hline$A$ & 2 & 1.017 & -0.56 & 0.994 & 0.38 \\
\hline Average $A$ & & & & 0.993 & 0.39 \\
\hline$B$ & 1 & 0.845 & -0.65 & 0.887 & 1.33 \\
\hline$B$ & 2 & 0.868 & -0.61 & 0.891 & 1.32 \\
\hline Average $B$ & & & & 0.889 & 1.33 \\
\hline C & 1 & 1.087 & -1.62 & 0.963 & 0.92 \\
\hline$C$ & 2 & 0.955 & 0.00 & 0.968 & 0.74 \\
\hline Average $C$ & & & & 0.966 & 0.83 \\
\hline $\begin{array}{l}\text { Overall } \\
\text { average }\end{array}$ & & 0.966 & -0.67 & 0.949 & 0.85 \\
\hline \multicolumn{6}{|l|}{ (B) } \\
\hline$A$ & 1 & 1.129 & -3.04 & 0.947 & 1.24 \\
\hline$A$ & 2 & 1.135 & -4.66 & 0.871 & 2.50 \\
\hline Average $A$ & & & & 0.909 & 1.87 \\
\hline$B$ & 1 & 0.003 & -5.57 & 0.004 & 2.55 \\
\hline$B$ & 2 & 0.176 & -7.52 & 0.154 & 3.71 \\
\hline Average $B$ & & & & 0.079 & 3.13 \\
\hline C & 1 & 0.189 & -3.70 & 0.135 & 4.54 \\
\hline$C$ & 2 & 0.558 & -4.96 & 0.431 & 3.83 \\
\hline Average $C$ & & & & 0.283 & 4.19 \\
\hline $\begin{array}{l}\text { Overall } \\
\text { average }\end{array}$ & & 0.532 & -4.91 & 0.424 & 3.06 \\
\hline
\end{tabular}

employed the TIFF formatted images in this study. Since no contrast enhancement was performed on the displayed images in the observer study, it is unlikely that a bit depth greater than 8 bits could have been perceived in the displayed images. Therefore, it is unlikely that compression to 8 bits influenced our results.

\section{Stereoscopic viewer and virtual cursor}

The images were displayed on a personal computer using a Model SS-03 Stereo Display Processor from Neotek, Inc. (Pittsburgh, PA). We used Neotek's Composer software to format the images and their optional Presenter software to display the images along with a virtual cursor. The Presenter software also generates a display of the $x, y$, and z-positions of the virtual cursor. The Neotek system produces stereo images via a method termed "synch-doubling." In this method, the left eye image is stored above the right eye image in the video graphics board (see Fig. 2), and an additional vertical synch pulse is inserted between the two images in the video signal coming from the computer. This synch-doubling causes the images to be displayed fully on the monitor at twice the normal refresh rate for reduced flicker (i.e., if the board is run at a $60 \mathrm{~Hz}$ refresh rate, the images are displayed at $120 \mathrm{~Hz}$ ). The graphics board was operated in the 1024 (horizontal) $\times 768$ (vertical) resolution mode recommended by Neotek. The Neotek Composer software downsized the $1024 \times 1024$ images to fit two images (the left on top of the right) within this resolution. That is, it converted the left and right eye images to each be about 


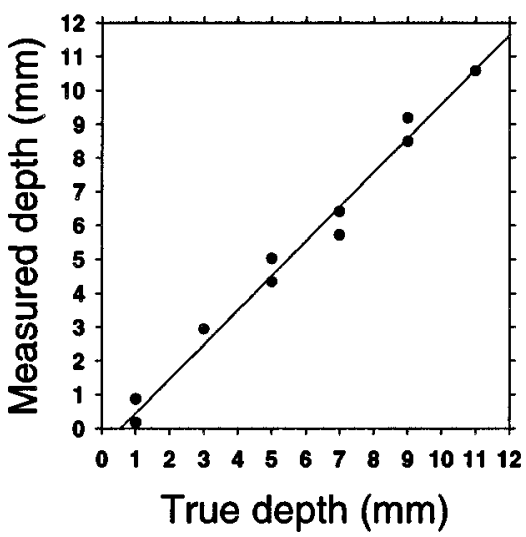

$1024 \times 340$. The loss in vertical resolution was necessitated by the synch-doubling. It had minimal effect in this study since the stereo shift direction corresponding with the horizontal display direction.

\section{Observer study}

Three observers (two medical physicists and a computer scientist) viewed the images on the computer monitor with a pair of Neotek stereo glasses. These employ LCD shutters that are synchronized with the display to allow viewing of the left image by the left eye and the right image by the right eye. The observers used the up and down arrow keys on the computer keyboard to adjust the position of a cross-shaped virtual cursor to best match the perceived location of each fibril, and noted the $z$ (depth) coordinates on a data sheet.

The $z$-coordinate was separately calibrated using the known positions of fibrils in the phantom. This was accomplished by viewing the images without the stereo glasses (see Fig. 2) and adjusting the left and right eye cursors to overlay the left and right eye representations of the vertical fibrils in the two images. The z-coordinates of the cursor were linearly fit to the known positions to obtain a calibration line. For the computations, the known depths were taken to be the known distances between the fibrils and the back surface of the phantom.

Each observer analyzed images of two configurations of the phantom. Thus, each observer made 50 vertical filament depth measurements and 50 horizontal filament depth measurements. Linear least-square fits were performed to compare the measurements with the true depths.

\section{RESULTS}

Table I lists the slopes, intercepts, correlation coefficients ( $r$-values) and standard errors of the estimates (SEE) of the least squares fits to the depth measurements made by the readers in each of the two images that they examined. The results in this table are separated into those for the vertically and horizontally oriented fibrils. Plots of the best and worst results in terms of the standard errors of the estimates are displayed in Figs. 3 and 4. Computed root mean square (RMS) errors for the fibril measurements are listed in Table II.

\section{DISCUSSION}

All observers could estimate the absolute depths of the vertically oriented objects fairly accurately in digital stereomammograms; however, only one observer was able to accurately estimate the depths of the horizontally oriented objects. For the vertically oriented fibrils, the overall average $r$-value was 0.949 and SEE was $0.85 \mathrm{~mm}$. The RMS errors in the depth measurements of the vertically oriented fibrils ranged from $0.6 \mathrm{~mm}$ to $1.9 \mathrm{~mm}$ with an average value for all three readers of $1.2 \mathrm{~mm}$. These RMS errors indicate that the absolute measurements with the virtual cursor can be accu-

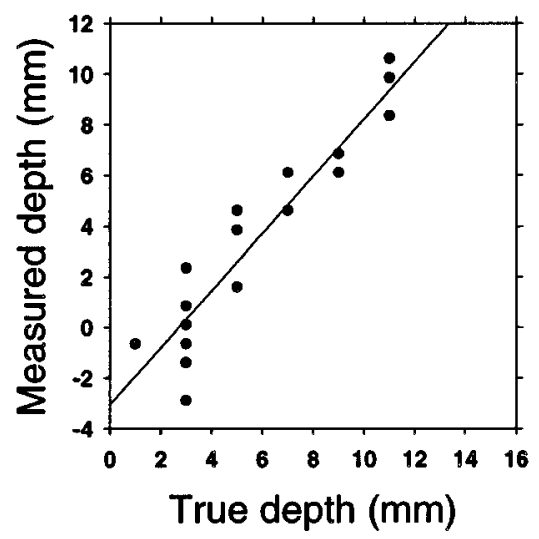

Medical Physics, Vol. 27, No. 6, June 2000

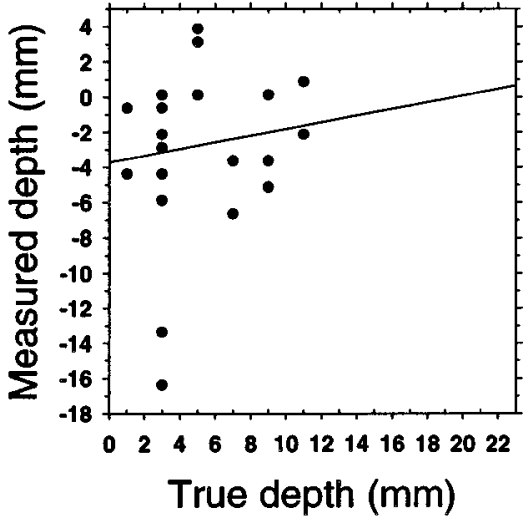

FIG. 4. Plots of the best and worst measured vs true depths of the horizontal fibrils for the three readers. The plot with the best accuracy $(r=0.947$, $\mathrm{SEE}=1.24 \mathrm{~mm}$ ) is shown on the left, and the plot with the worst accuracy $(r=0.135, \mathrm{SEE}=4.54 \mathrm{~mm})$ is shown on the right. 
TABLE II. Root mean square (RMS) errors of measured depths of fibrils. ${ }^{a}$ (A) Vertically oriented fibrils. (B) Horizontally oriented fibrils.

\begin{tabular}{ccc}
\hline \hline Reader & Image & RMS error $(\mathrm{mm})$ \\
\hline (A) & 1 & \\
$A$ & 2 & 0.59 \\
$A$ & 1 & 0.62 \\
$B$ & 2 & 1.91 \\
$B$ & 1 & 1.78 \\
$C$ & 2 & 1.53 \\
$C$ & & 0.75 \\
Overall average & & 1.20 \\
$(\mathrm{~B})$ & 1 & \\
$A$ & 2 & 2.70 \\
$A$ & 1 & 4.76 \\
$B$ & 2 & 11.36 \\
$B$ & 1 & 12.56 \\
$C$ & 2 & 9.30 \\
$C$ & & 8.24 \\
Overall Average & & 8.15 \\
\hline \hline
\end{tabular}

${ }^{\mathrm{a}} \mathrm{RMS}$ error $\left.=\sqrt{\sum_{i=1}^{25}\left(\text { true depth }_{i}-\text { measured depth }\right.}\right)^{2} / 25$.

rate to within $2 \mathrm{~mm}$. This is consistent with relative stereoscopic studies performed by Doi and Duda ${ }^{26}$ and Higashida et al. ${ }^{15}$ and absolute stereoscopic studies performed by Fencil et al. ${ }^{16}$ Doi and Duda and Higashida et al. investigated observers' abilities to distinguish (as opposed to measure) the separation of objects that were superimposed on stepwedge phantoms. In Doi and Duda's study, ${ }^{26}$ the objects were $0.2 \mathrm{~mm}$ diam aluminum wires. A matrix of "plus" objects were formed by placing horizontally and vertically oriented pieces of wire at the bottom of the stepwedge, with their counterparts located directly above on the step of known thickness (e.g., horizontal wire on step if bottom wire is vertical). The "plus" objects were imaged stereoscopically using a geometric magnification factor of 2 and x-ray focal spot shifts of $1.25 \%, 2.5 \%$, and $5 \%$ of the focus-to-film distance. These investigators found that observers could correctly identify $1 \mathrm{~mm}$ separations between two aluminum wires $80 \%$ of the time for the $5 \%$ tube shift and between 60 and $70 \%$ for the other tube shifts. In Hagashida et al.'s study, ${ }^{15}$ a similar phantom was used. Teflon tube objects filled with contrast media were arranged on the stepwedge to form cross (or " $X$ ") shaped objects. They employed a geometric magnification factor of 1.1 and the $\mathrm{x}$-ray focal spot shift for stereoscopic imaging was $6.5 \%$ of the focus-toimage intensifier distance. They found that observers could correctly identify $1.6 \mathrm{~mm}$ separations between $1 \mathrm{~mm}$ diameter tubes containing $25 \%$ iodine contrast more than $80 \%$ of the time.

Our study and results can be distinguished from those of Doi and Duda ${ }^{26}$ and Higashida et al. ${ }^{15}$ because we investigated absolute rather than relative stereoscopic measurements and because we analyzed horizontally and vertically oriented objects separately. With respect to absolute measurements, Fencil et al. ${ }^{16}$ imaged a box phantom containing aluminum wires that simulated blood vessels at different angles. Fencil et al. employed an automated crosscorrelation technique to determine the position of a wire (vessel) segment in the second image of a stereoscopic pair after it was selected in the first image. They obtained an average calculated distance error of approximately $\pm 2 \mathrm{~mm}$, which is similar to our error for vertically oriented fibrils and the errors in the studies of Doi and Duda and Higashida et al. that are cited above. Our technique is easier to implement than Fencil et al.'s but more observer dependent, as the observer effectively judges the correlation between the cursor and the fibrils in both images of the stereoscopic pair.

The fact that the observers in our study did worse at measuring the depths of horizontally as opposed to vertically oriented fibrils is not surprising since the image discrepancy and hence the stereoscopic effect is less for the horizontal objects (i.e., there is considerable overlap between corresponding horizontally oriented objects in the stereo pairs, and the shifts in positions are not as apparent). ${ }^{14}$ There appears to be a larger difference in the performance among the observers for the horizontally oriented fibrils, with one observer performing very well and the other two very poorly. This may be related to different aptitudes for stereoscopic visualization.

The orientations of most objects in actual mammograms are combinations of horizontal and vertical. Further studies are planned to evaluate absolute depth measurements of fibrils oriented at various intermediate angles and of objects of different shapes. The effects of the shape and contrast of the virtual cursor and of the stereo shift angle on the accuracy of the depth measurements will also be investigated. Finally, the cursors will be applied to digital stereomammograms of breast biopsy samples to determine their applicability in estimating lesion depths and dimensions and in providing additional depth cues for improved stereoscopic image interpretation.

\section{ACKNOWLEDGMENTS}

This work is supported by U.S. Army Medical Research and Materiel Command Grant DAMD 17-98-1-8210. The content of this publication does not necessarily reflect the position of the funding agency, and no official endorsement of any equipment and product of any companies mentioned in this publication should be inferred.

\footnotetext{
${ }^{a}$ Send all correspondences to the following address: Department of Radiology, University of Michigan Hospitals, Room B1 F510C, 1500 East Medical Center Drive, Ann Arbor, Michigan 48109-0030. Office: 734936-7474; Fax: 734-936-7948; electronic mail: goodsitt@umich.edu

${ }^{1}$ H. Seidman, S. K. Gelb, E. Silverberg, N. LaVerda, and J. A. Lubera, "Survival experience in the Breast Cancer Detection Demonstration Project,"' CA-Cancer J. Clin. 37, 258-290 (1987).

${ }^{2}$ M. Moskowitz, Benefit and Risk, in Breast Cancer Detection: Mammography and Other Methods in Breast Imaging, 2nd ed., edited by L. W. Bassett and R. H. Gold (Grune and Stratton, New York, 1987).

${ }^{3}$ J. E. Martin, M. Moskowitz, and J. R. Milbrath, "Breast cancer missed by mammography,'” AJR, Am. J. Roentgenol. 132, 737-739 (1979).

${ }^{4}$ B. J. Hillman et al., "Mammogram interpretation by physician assistants," AJR, Am. J. Roentgenol. 149, 907-911 (1987).

${ }^{5} \mathrm{~L}$. Kalisher, "Factors influencing false negative rates in xeromammography," Radiology 133, 297-301 (1979).
} 
${ }^{6}$ L. W. Bassett, D. H. Bunnell, R. Jahanshahi, R. H. Gold, R. D. Arndt, and J. Linsman, 'Breast cancer detection: One vs two views,'” Radiology 165, 95-97 (1987).

${ }^{7}$ C. J. Baines et al., "Sensitivity and specificity of first screen mammography in the Canadian National Breast Screening Study: A preliminary report from five centers,', Radiology 160, 295-298 (1986).

${ }^{8}$ P. J. Haug, I. M. Tocino, P. D. Clayton, and T. L. Bair, "Automated management of screening and diagnostic mammography," Radiology 164, 747-752 (1987).

${ }^{9}$ M. G. Wallis, M. T. Walsh, and J. R. Lee, “A review of false negative mammography in a symptomatic population,' Clin. Radiol. 44, 13-15 (1991).

${ }^{10}$ J. A. Harvey, L. L. Fajardo, and C. A. Innis, "Previous mammograms in patients with impalpable breast carcinomas: Retrospective vs blinded interpretation,' AJR, Am. J. Roentgenol. 161, 1167-1172 (1993).

${ }^{11}$ R. E. Bird, T. W. Wallace, and B. C. Yankaskas, "Analysis of cancers missed at screening mammography," Radiology 184, 613-617 (1992).

${ }^{12}$ V. P. Jackson, R. E. Hendrick, S. A. Feig, and D. B. Kopans, “'Imaging of the radiographically dense breast,' Radiology 188, 297-301 (1993).

${ }^{13}$ S. L. Warren, "Roentgenologic study of the breast,' AJR, Am. J. Roentgenol. 24, 113-124 (1930)

${ }^{14}$ T. S. Curry, J. E. Dowdey, and R. C. Murry, Christensen's Physics of Diagnostic Radiology, 4th ed. (Lea \& Febiger, Philadelphia, 1990).

${ }^{15}$ Y. Higashida, Y. Hirata, R. Saito, S. Doudajuki, H. Bussaka, and M. Takahashi, "Depth determination on sterescopic digital subtraction angiograms,'” Radiology 168, 560-562 (1988).

${ }^{16}$ L. E. Fencil, K. Doi, and K. R. Hoffman, "Accurate analysis of blood vessel sizes and stenotic lesions using stereoscopic DSA system,' Invest. Radiol. 23, 33-41 (1988).

${ }^{17}$ T. Moll, P. Douek, G. Finet, F. Turjman, C. Picard, D. Revel, and M.
Amiel, "Clinical assessment of a new stereoscopic digital angiography system,' Cardiovasc. Intervent. Radiol. 21, 11-16 (1998).

${ }^{18}$ J. Hsu, D. M. Chelberg, C. F. Babbs, Z. Pizlo, and E. Delp, 'Preclinical ROC studies of digital stereomammography,' IEEE Trans. Med. Imaging 14, 318-327 (1995).

${ }^{19}$ D. Getty, "'Stereoscopic digital mammography: Improving diagnostic accuracy," Technology Transfer Workshop on Breast Cancer Detection, Diagnosis, and Treatment, May 1-2, 1997, Washington, D. C., 1997.

${ }^{20}$ R. E. Hendrick and S. H. Parker, "Stereotaxic Imaging," in Syllabus: A Categorical Course in Physics Technical Aspects of Breast Imaging, 3rd ed. (Radiological Society of North America, Inc., Northbrook, 1994), pp. 263-274.

${ }^{21}$ D. R. W. Butts and D. F. McAllister, "Implementation of true 3D cursors in computer graphics,' Proc. SPIE 902, 74-82 (1988).

${ }^{22}$ F. W. Reinhardt, "Effects of Depth Cues on Judgements Using a FieldSequential Stereoscopic CRT Display,' Ph.D. dissertation, Industrial Engineering and Operations Research Department, Virginia Polytechnic Institute and State University, 1990.

${ }^{23}$ Y. Y. Yeh and L. D. Silverstein, "Depth discrimination in stereoscopic displays,', SID '89 20, 372-375 (1989).

${ }^{24}$ D. Drascic and P. Milgram, "Positioning accuracy of a virtual stereographic pointer in a real stereoscopic video world,', SPIE 1457, 58-69 (1991).

${ }^{25}$ S. Zhai, W. Buxton, and P. Milgram, "The partial occlusion effect: Utilizing semitransparency in 3D human computer interaction,' in Proceedings of the CHI'94, ACM Conference on Human Factors in Computing Systems, April, 1994, Boston, MA, 1994 (unpublished).

${ }^{26}$ K. Doi and E. E. Duda, "Detectability of depth information by use of magnification stereoscopic technique in cerebral angiography,' Radiology 146, 91-95 (1983). 